\title{
Application of spectral envelope functions of multilayer structures for analytical determination of antireflection conditions
}

\author{
Kushnir O.P. \\ Department of Physics, Lviv National Agrarian University, 1 V. Velykogo St., \\ Dublyany, 80381 Lviv region, Ukraine, E-mail: o-p-ku@ukr.net
}

Received: 06.02.2009

\begin{abstract}
Envelope functions for the reflection and transmission spectra of multilayer structures are determined. Analytical expressions for the phase thicknesses of two arbitrary layers ensuring zero reflectance for the whole transparent structure are found with the aid of those envelopes.
\end{abstract}

Keywords: antireflection conditions, reflection spectra envelopes, transmission spectra envelopes, multilayer coatings.

PACS: $42.79 . \mathrm{Wc} ; 78.20$.

UDC: 535.312

\section{Introduction}

Envelopes of reflection and transmission spectra of one-layer systems have been used by many researches in order to derive optical constants of those systems [1-7]. In particular, the authors $[8,9]$ have employed the envelope function for spectral reflection minima for finding of expressions for the incidence angles that provide zero $p$ - or $s$-reflectance of one-layer structures. In spite of a common opinion that finding out the analytical functions for the spectral envelopes is hardly possible, even for relatively simple case of two transparent films [10], analytical expressions for the envelopes of both transparent and absorbing two- and three-layer structures have nevertheless been obtained [11].

In this study we present expressions for the envelopes of reflection and transmission spectra of multilayer structures. These results are further applied for ascertaining analytical antireflection conditions for transparent multilayer structures. It has turned out that the antireflection conditions derived by us generalise the corresponding conditions for the case of two-layer coatings [12,13], which have been earlier obtained with the aid of other techniques.

\section{Envelope functions for the reflection and transmission spectra of absorbing multilayer structures}

Let us consider a multilayer system consisting of $k$ isotropic layers with the phase thicknesses $\quad \tilde{\delta}_{v}=\frac{2 \pi d_{v} \tilde{n}_{v}}{\lambda} \cos \tilde{\beta}_{v} \quad(v=1,2, \ldots, k)$. Here $d_{v} \quad$ and $\tilde{n}_{v}=n_{v}-i \kappa_{v}$ denote 
respectively the thicknesses and the complex refractive indices of the layers and $\tilde{\beta}_{v}$ is the angle of incidence at the boundary of $v /(v+1)$ media.

The medium above the layered system is semi-infinite, with the refractive index $n_{0}$, and there is a semi-infinite substrate with the refractive index $n_{k+1}$ below the system. The amplitude reflectance and transmittance of the multilayer structure may be written respectively as [14]

$$
\begin{aligned}
& \tilde{r}_{0, k+1}=\frac{\tilde{r}_{0, s}+\tilde{r}_{s, k+1} \tilde{h}_{0, s} e^{-2 i \tilde{\delta}_{s}}}{1-\tilde{r}_{s, 0} \tilde{r}_{s, k+1} e^{-2 i \tilde{\delta}_{s}}}, \\
& \tilde{t}_{0, k+1}=\frac{\tilde{t}_{0, s} \tilde{t}_{s, k+1} e^{-i \tilde{\delta}_{s}}}{1-\tilde{r}_{s, 0} \tilde{r}_{s, k+1} e^{-2 i \tilde{\delta}_{s}}},
\end{aligned}
$$

where $s$ is an arbitrary number $(0<s<k+1)$, the complex parameter $\tilde{h}_{j, u}=\chi_{j, u} e^{i \gamma_{j, u}}$ is defined as $\tilde{h}_{j, u}=\tilde{t}_{j, u} \tilde{t}_{u, j}-\tilde{r}_{j, u} \tilde{r}_{u, j}(j=0,1, \ldots, k+1$ and $u=0,1, \ldots, k+1)$ and the complex amplitude reflectance $\left(\tilde{r}_{j, u}=\sigma_{j, u} e^{i \phi_{j, u}}\right)$ and transmittance $\left(\tilde{t}_{j, u}=\tau_{j, u} e^{i \theta_{j, u}}\right)$ are determined according to the well-known Fresnel formulae. Namely, we have $\tilde{r}_{j, u}=-\frac{\sin \left(\tilde{\beta}_{j}-\tilde{\beta}_{u}\right)}{\sin \left(\tilde{\beta}_{j}+\tilde{\beta}_{u}\right)}, \tilde{t}_{j, u}=\frac{2 \cos \tilde{\beta}_{j} \sin \tilde{\beta}_{u}}{\sin \left(\tilde{\beta}_{j}+\tilde{\beta}_{u}\right)}$ for the $s$-polarization and $\tilde{r}_{j, u}=\frac{\tan \left(\tilde{\beta}_{j}-\tilde{\beta}_{u}\right)}{\tan \left(\tilde{\beta}_{j}+\tilde{\beta}_{u}\right)}$, $\tilde{t}_{j, u}=\frac{2 \cos \tilde{\beta}_{j} \sin \tilde{\beta}_{u}}{\sin \left(\tilde{\beta}_{j}+\tilde{\beta}_{u}\right) \cos \left(\tilde{\beta}_{j}-\tilde{\beta}_{u}\right)}$ for the $p$-polarization in the case of $|u-j|=1$, or $\tilde{r}_{j, u}=\frac{\tilde{M}_{j, u, 21}}{\tilde{M}_{j, u, 11}}[15]$ and $\tilde{t}_{j, u}=\frac{\tilde{t}_{j, j \pm 1} \prod_{v=j \pm 1}^{u \mp 1}\left(\tilde{t}_{v, v \pm 1} e^{-i \tilde{\delta}_{v}}\right)}{\tilde{M}_{j, u, 11}}[11]$ in the case of $|u-j|>1$. The complex parameters $\tilde{M}_{j, u, 11}$ and $\tilde{M}_{j, u, 21}$ could be calculated using the following $2 \times 2$ matrix multiplication:

$$
\begin{aligned}
& \left(\begin{array}{ll}
\tilde{M}_{j, u, 11} & \tilde{M}_{j, u, 12} \\
\tilde{M}_{j, u, 21} & \tilde{M}_{j, u, 22}
\end{array}\right)=\left(\begin{array}{cc}
1 & \tilde{r}_{j, j \pm 1} \\
\tilde{r}_{j, j \pm 1} & 1
\end{array}\right) \times\left(\begin{array}{cc}
1 & \tilde{r}_{j \pm 1, j \pm 2} \\
\tilde{r}_{j \pm 1, j \pm 2} e^{-2 i \tilde{\delta}_{j \pm 1}} & e^{-2 i \tilde{\delta}_{j \pm 1}}
\end{array}\right) \\
& \times\left(\begin{array}{cc}
1 & \tilde{r}_{j \pm 2, j \pm 3} \\
\tilde{r}_{j \pm 2, j \pm 3} e^{-2 i \tilde{\delta}_{j \pm 2}} & e^{-2 i \tilde{\delta}_{j \pm 2}}
\end{array}\right) \times \cdots \times\left(\begin{array}{cc}
1 & \tilde{r}_{u \mp 1, u} \\
\tilde{r}_{u \mp 1, u} e^{-2 i \tilde{\delta}_{u \mp 1}} & e^{-2 i \tilde{\delta}_{u \mp 1}}
\end{array}\right)
\end{aligned}
$$

Hear the upper signs in " \pm " and " $\mp "$ refer to the condition $u>j+1$ and the lower ones to the condition $j>u+1$. 
On the basis of Eqs. (1) and (2) the reflectance $R_{0, k+1}=\tilde{r}_{0, k+1} \tilde{r}_{0, k+1}^{*}$ and the transmittance $T_{0, k+1}=\frac{n_{k+1}}{n_{0}} \frac{\cos \beta_{k+1}}{\cos \beta_{0}} \tilde{t}_{0, k+1} \tilde{t}_{0, k+1}^{*}$ may be represented as

$$
\begin{aligned}
R_{0, k+1}= & \left.\frac{R_{s}^{\min }+\frac{4 \sigma_{0, s} \sigma_{s, k+1} \chi_{0, s} \Omega_{s}}{\left(1-\sigma_{s, 0} \sigma_{s, k+1} \Omega_{s}\right)^{2}} \cos ^{2}\left(\frac{2 \operatorname{Re} \tilde{\delta}_{s}+\phi_{0, s}-\phi_{s, k+1}-\gamma_{0, s}}{2}\right)}{1+\frac{4 \sigma_{s, 0} \sigma_{s, k+1} \Omega_{s}}{\left(1-\sigma_{s, 0} \sigma_{s, k+1} \Omega_{s}\right)^{2}} \sin ^{2}\left(\frac{2 \operatorname{Re} \tilde{\delta}_{s}-\phi_{s, 0}-\phi_{s, k+1}}{2}\right)}\right) \\
= & \frac{R_{s}^{\max }-\frac{4 \sigma_{0, s} \sigma_{s, k+1} \chi_{0, s} \Omega_{s}}{\left(1+\sigma_{s, 0} \sigma_{s, k+1} \Omega_{s}\right)^{2}} \sin ^{2}\left(\frac{2 \operatorname{Re} \tilde{\delta}_{s}+\phi_{0, s}-\phi_{s, k+1}-\gamma_{0, s}}{2}\right)}{1-\frac{4 \sigma_{s, 0} \sigma_{s, k+1} \Omega_{s}}{\left(1+\sigma_{s, 0} \sigma_{s, k+1} \Omega_{s}\right)^{2}} \cos ^{2}\left(\frac{2 \operatorname{Re} \tilde{\delta}_{s}-\phi_{s, 0}-\phi_{s, k+1}}{2}\right)} \\
T_{0, k+1}= & \frac{1-\frac{4 \sigma_{s, 0} \sigma_{s, k+1} \Omega_{s}}{\left(1+\sigma_{s, 0} \sigma_{s, k+1} \Omega_{s}\right)^{2}} \cos ^{2}\left(\frac{2 \operatorname{Re} \tilde{\delta}_{s}-\phi_{s, 0}-\phi_{s, k+1}}{2}\right)}{1+\frac{4 \sigma_{s, 0} \sigma_{s, k+1} \Omega_{s}}{\left(1-\sigma_{s, 0} \sigma_{s, k+1} \Omega_{s}\right)^{2}} \sin ^{2}\left(\frac{\left.2 \operatorname{Re} \tilde{\delta}_{s}-\phi_{s, 0}-\phi_{s, k+1}\right)}{2}\right)}
\end{aligned}
$$

where $\Omega_{s}=e^{2 \operatorname{Im} \tilde{\delta}_{s}}$ and

$$
\begin{aligned}
& R_{s}^{\min , \max }=\left(\frac{\sigma_{0, s} \mp \sigma_{s, k+1} \chi_{0, s} \Omega_{s}}{1 \mp \sigma_{s, 0} \sigma_{s, k+1} \Omega_{s}}\right)^{2}, \\
& T_{s}^{\min , \max }=\frac{n_{k+1}}{n_{0}} \frac{\cos \beta_{k+1}}{\cos \beta_{0}} \frac{\tau_{0, s}^{2} \tau_{s, k+1}^{2} \Omega_{s}}{\left(1 \pm \sigma_{s, 0} \sigma_{s, k+1} \Omega_{s}\right)^{2}} .
\end{aligned}
$$

Notice that here the sign "+" refers to $R_{s}^{\max }$ and $T_{s}^{\min }$ and "-" to $R_{s}^{\min }$ and $T_{s}^{\max }$.

Using standard computer modelling techniques, we have shown that the functions $R_{s}^{\min , \max }$ and $T_{s}^{\min , \max }$ are the envelope functions of the reflection and transmission spectra provided that the relation

$$
d_{s} n_{s}>d_{v} n_{v} \quad(v=1,2, \ldots, s-1, s+1, s+2, \ldots, k)
$$

holds true for the optical thickness of the layer with the number $s$ (see Fig. 1). Regardless of whether the optical thickness of that arbitrary layer satisfies the condition (6) or not, the envelope function for the minima of the reflection spectrum $R_{s}^{\min }$ given by Eq. (4) may be successfully used for establishing antireflection conditions for the transparent multilayer structure. 


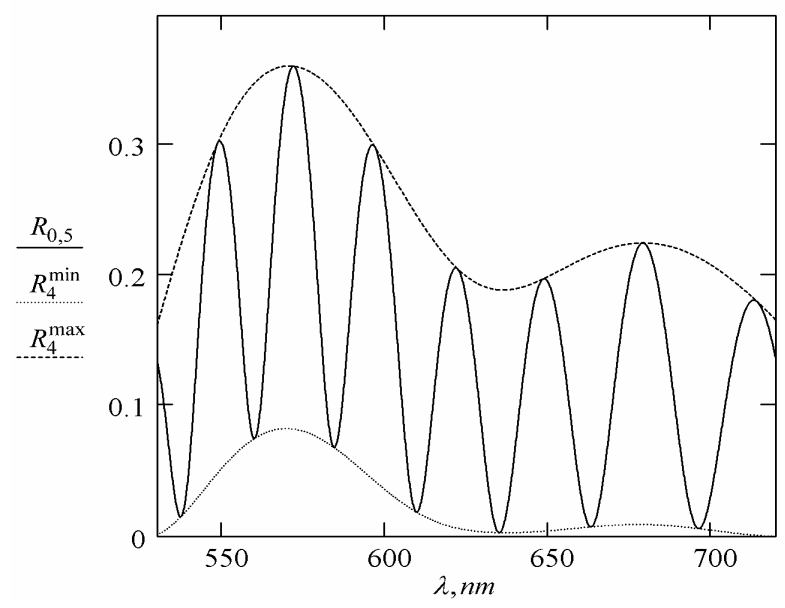

Fig. 1. Computed reflectance $R_{0,5}$ of four-layer coating and envelopes $R_{4}^{\min , \max }$ given by Eq. (4) at the normal incidence. The complex refractive indices and the thicknesses of layers are chosen as follows: $n_{0}=1, \quad \tilde{n}_{1}=1.5-0.001 i$, $\tilde{n}_{2}=2.1-0.002 i, \quad \tilde{n}_{3}=1.8-0.003 i$, $\tilde{n}_{4}=2.3-0.005 i$ and $n_{5}=4.0$; $d_{1}=0.4 \mu \mathrm{m}, \quad d_{2}=0.35 \mu \mathrm{m}$, $d_{3}=0.2 \mu \mathrm{m}$ and $d_{4}=2.5 \mu \mathrm{m}$.

\section{Antireflection conditions for the transparent multilayer structure}

Basing on Eqs. (3) and (4), one can conclude that the condition $R_{0, k+1}=0$ is satisfied only if the envelope of the reflection minima $R_{s}^{\min }$ (for an arbitrary number $s$ ranging as $0<s<k+1$ ) equals to zero. In its turn, the condition $R_{s}^{\min }=0$ fulfils if

$$
\sigma_{0, s}-\sigma_{s, k+1}=0
$$

or

$$
\sigma_{0, s}^{2}-\tilde{r}_{s, k+1} \tilde{r}_{s, k+1}^{*}=0 .
$$

Here we have taken into consideration that $\chi_{j, u}=1$ [16] and $\Omega_{s}=1$ for the transparent structures.

Having expressed the amplitude reflectance given by Eq. (1) for the structure $s, s+1, \ldots, k, k+1$,

$$
\tilde{r}_{s, k+1}=\frac{\tilde{r}_{s, m}+\tilde{r}_{m, k+1} \tilde{h}_{s, m} e^{-2 i \delta_{m}}}{1-\tilde{r}_{m, s} \tilde{r}_{m, k+1} e^{-2 i \delta_{m}}}
$$

(with $m$ representing an arbitrary number from the interval $s<m<k+1$ ), we are able to solve Eqs. (7):

$$
\tan \delta_{m}^{ \pm}=\frac{2 \sigma_{s, m} \sigma_{m, k+1} \sin \left(\phi_{s, m}-\phi_{m, k+1}-\gamma_{s, m}\right)-2 \sigma_{m, s} \sigma_{m, k+1} \sigma_{0, s}^{2} \sin \left(\phi_{m, s}+\phi_{m, k+1}\right) \pm \sqrt{Q}}{\left(\sigma_{s, m}+\sigma_{0, s}-\sigma_{m, s} \sigma_{m, k+1} \sigma_{0, s}+\sigma_{m, k+1}\right)\left(\sigma_{s, m}-\sigma_{0, s}+\sigma_{m, s} \sigma_{m, k+1} \sigma_{0, s}+\sigma_{m, k+1}\right)-G},
$$

where

$$
\begin{aligned}
Q & =-\left(\sigma_{s, m}+\sigma_{0, s}-\sigma_{m, s} \sigma_{m, k+1} \sigma_{0, s}-\sigma_{m, k+1}\right)\left(\sigma_{s, m}-\sigma_{0, s}+\sigma_{m, s} \sigma_{m, k+1} \sigma_{0, s}-\sigma_{m, k+1}\right) \\
& \times\left(\sigma_{s, m}-\sigma_{0, s}-\sigma_{m, s} \sigma_{m, k+1} \sigma_{0, s}+\sigma_{m, k+1}\right) \\
& \times\left(\sigma_{s, m}+\sigma_{0, s}+\sigma_{m, s} \sigma_{m, k+1} \sigma_{0, s}+\sigma_{m, k+1}\right)+16 \sigma_{s, m} \sigma_{m, s} \sigma_{m, k+1}^{2} \sigma_{0, s}^{2} \cos ^{2}\left(\frac{\phi_{s, m}+\phi_{m, s}-\gamma_{s, m}}{2}\right),
\end{aligned}
$$




$$
G=4 \sigma_{m, k+1} \sigma_{s, m} \cos ^{2}\left(\frac{\phi_{s, m}-\phi_{m, k+1}-\gamma_{s, m}}{2}\right)+4 \sigma_{m, s} \sigma_{m, k+1} \sigma_{0, s}^{2} \cos ^{2}\left(\frac{\phi_{m, s}+\phi_{m, k+1}}{2}\right) .
$$

If the antireflection condition given by Eq. (7) is rewritten in the form

$$
\tilde{r}_{0, m} \tilde{r}_{0, m}^{*}-\sigma_{m, k+1}^{2}=0
$$

where the amplitude reflectance (1) is $\tilde{r}_{0, m}=\frac{\tilde{r}_{0, s}+\tilde{r}_{s, m} \tilde{h}_{0, s} e^{-2 i \delta_{s}}}{1-\tilde{r}_{s, 0} \tilde{r}_{s, m} e^{-2 i \delta_{s}}}$ for the structure $0,1, \ldots, m-1, m$ (the case of $s<m$ ), then the solution of Eq. (9) is as follows:

$$
\tan \delta_{s}^{\mp}=\frac{2 \sigma_{0, s} \sigma_{s, m} \sin \left(\phi_{s, m}-\phi_{0, s}+\gamma_{0, s}\right)+2 \sigma_{0, s} \sigma_{m, s} \sigma_{m, k+1}^{2} \sin \left(\gamma_{0, s}+\gamma_{s, m}-\phi_{m, s}-\phi_{0, s}\right) \mp \sqrt{Q}}{-\left(\sigma_{s, m}+\sigma_{0, s}-\sigma_{m, s} \sigma_{m, k+1} \sigma_{0, s}+\sigma_{m, k+1}\right)\left(\sigma_{s, m}+\sigma_{0, s}+\sigma_{m, s} \sigma_{m, k+1} \sigma_{0, s}-\sigma_{m, k+1}\right)+W},
$$

where $W=4 \sigma_{s, m} \sigma_{0, s} \cos ^{2}\left(\frac{\phi_{s, m}-\phi_{0, s}+\gamma_{0, s}}{2}\right)+4 \sigma_{m, s} \sigma_{m, k+1}^{2} \sigma_{0, s} \cos ^{2}\left(\frac{\gamma_{0, s}+\gamma_{s, m}-\phi_{m, s}-\phi_{0, s}}{2}\right)$.

Eq. (8) for the phase thicknesses $\delta_{m}^{+}$and Eq. (10) for $\delta_{s}^{-}$or the corresponding relations for $\delta_{m}^{-}$and $\delta_{s}^{+}$provide zero $p$ - or $s$-reflectance in the case of oblique incidence and the antireflection condition in the case of normal incidence. Here $s$ and $m$ are the numbers of two arbitrary layers $(s<m)$. It is also worth mentioning that the antireflection is possible only for those transparent structures, of which parameters satisfy the condition $Q \geq 0$.

\section{Numerical example}

As an example, let us derive the antireflection conditions at a given wavelength for a transparent ten-layer structure in case of the normal incidence. Let us this structure in the air $\left(n_{0}=1\right)$ be deposited on germanium substrate $\left(n_{k+1}=n_{11}=4.0\right)$. The two solutions for the phase thicknesses of the second and eighth layers, whose numbers are taken arbitrarily, have been found out for arbitrarily chosen refractive indices of all the layers $\left(n_{1}=1.37, n_{2}=1.43, n_{3}=1.55, n_{4}=1.77, n_{5}=1.97, n_{6}=2.10, n_{7}=2.30, n_{8}=2.47\right.$, $n_{9}=2.77$ and $\left.n_{10}=2.96\right)$ and for arbitrarily chosen phase thicknesses of the eight layers $\left(\delta_{1}=1.755 \mathrm{rad}, \quad \delta_{3}=1.640 \mathrm{rad}, \quad \delta_{4}=1.582 \mathrm{rad}, \quad \delta_{5}=1.524 \mathrm{rad}, \quad \delta_{6}=1.466 \mathrm{rad}\right.$, $\delta_{7}=1.409 \mathrm{rad}, \delta_{9}=1.293 \mathrm{rad}$ and $\delta_{10}=1.236 \mathrm{rad}$ ) with the aid of Eqs. (8) and (10):

(1) $\delta_{2}=1.232 \mathrm{rad}, \delta_{8}=0.871 \mathrm{rad}$;

(2) $\delta_{2}=0.817 \mathrm{rad}, \delta_{8}=1.255 \mathrm{rad}$.

The calculation for the reflectance versus normalized frequency $\lambda_{0} / \lambda$ (with $\lambda_{0}$ being the wavelength that corresponds to the calculated phase thicknesses) is illustrated in Fig. 2. 


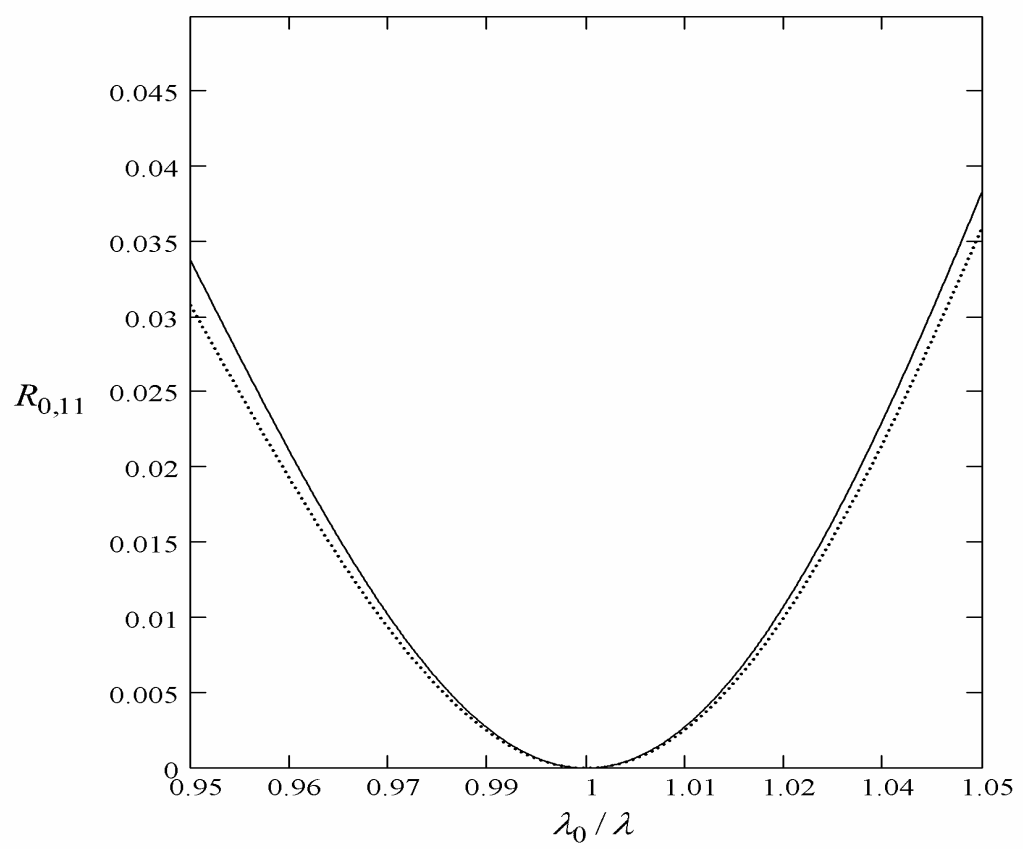

Fig. 2. Computed reflectance $R_{0,11}$ versus normalized frequency $\lambda_{0} / \lambda$ for the ten-layer coating. The refractive indices of the layers and their phase thicknesses at $\lambda_{0}$ are as follows: $n_{1}=1.37, n_{2}=1.43, n_{3}=1.55, n_{4}=1.77$, $n_{5}=1.97, \quad n_{6}=2.10, \quad n_{7}=2.30, \quad n_{8}=2.47, \quad n_{9}=2.77 \quad n_{10}=2.96 ;$ $\delta_{1}=1.755 \mathrm{rad}, \quad \delta_{3}=1.640 \mathrm{rad}, \quad \delta_{4}=1.582 \mathrm{rad}, \quad \delta_{5}=1.524 \mathrm{rad}$, $\delta_{6}=1.466 \mathrm{rad}, \quad \delta_{7}=1.409 \mathrm{rad}, \quad \delta_{9}=1.293 \mathrm{rad}, \quad \delta_{10}=1.236 \mathrm{rad} ;$ $\delta_{2}=1.232 \mathrm{rad}, \delta_{8}=0.871 \mathrm{rad}$ (solid curve), $\delta_{2}=0.817 \mathrm{rad}, \delta_{8}=1.255 \mathrm{rad}$ (dotted curve).

\section{Conclusions}

Utilisation of the functions of minimums envelope $R_{s}^{\min }$ has allowed us to find successfully the analytical conditions for the zero reflectance of multilayer coatings at a single light wavelength. These conditions might be reduced to determination of phase thicknesses of arbitrary two layers with the aid of Eqs. (8) and (10), provided that the refractive indices and the phase thicknesses of all the other layers satisfy the condition $Q \geq 0$. It is shown that the antireflection does not occur in case when $Q<0$.

The antireflection conditions revealed by us turn out to be more general when compare with those obtained with the other known methods for achieving zero reflectance at a single wavelength [17]. Namely, they allow choosing arbitrary refractive indices and phase thicknesses of all the layers under study, except for two arbitrary ones, of which the parameters should be thoroughly calculated.

The envelope functions for the reflection and transmission spectra determined in this work may be also used for many other purposes, including those mentioned above. 
Kushnir O.P.

\section{References}

1. Minkov D A, 2001. Method for determining the optical constants of thin dielectric films with variable thickness using only their shrunk reflection spectra. J. Phys. D: Appl. Phys. 34: 2489-2496.

2. Minkov D A, 1989. Calculation of the optical constants of a thin layer upon a transparent substrate from the reflection spectrum. J. Phys. D: Appl. Phys. 22: 1157-1161.

3. Kushev D B and Zheleva N N, 1995. Transmittivity, reflectivities and absorptivities of a semiconductor film with a linear variation in thickness. J. Phys. D: Appl. Phys. 28: 1239-1243.

4. Kushev D B, Zheleva N N, Gyulmezov M I and Koparanova M H, 1993. An envelope method for determination of the optical constants of absorptive films on absorptive substrates. Infrared Phys. 34: 163-167.

5. Swanepoel R, 1984. Determination of surface roughness and optical constants of inhomogeneous amorphous silicon films. J. Phys. E: Sci. Instrum. 17: 896-903.

6. Caricato A P, Fazzi A and Leggieri G, 2005. A computer program for determination of thin films thickness and optical constants. Appl. Surf. Sci. 248: 440-445.

7. Minkov D A, 1989. Method for determining the optical constants of a thin film on a transparent substrate. J. Phys. D: Appl. Phys. 22: 199-205.

8. Kosobutskyy P S and Kushnir O P, 2007. Regularities of manifestation of the Brewster and pseudo-Brewster angular conditions in spectra of light reflection from a thin transparent layer. Ukr. J. Phys. 52: 225-228.

9. Kosobutskyy P S and Kushnir O P, 2008. Regularities of oblique light reflection by a film caused by a multibeam interference. J. Phys. Stud. 12: 1701-1-1701-5.

10.Filippov V V, 2006. Envelope technique for the studies of two-film system lying on reflecting substrate. Opt. Spektrosk. 101: 485-489.

11.Kosobutskyy P S and Kushnir O P, 2008. Envelopes of optical interference spectra for duplex and triplex layered structures. Ukr. J. Phys. Opt. 9: 73-81.

12.Schuster K, 1949. Anwendung der Vierpoltheorie auf die Probleme der optischen Reflexionsminderung, Reflexionsverstarkung und der Interferenzfilter. Ann. Phys. 4: 352-356.

13.Catalan L A, 1962. Some computed optical properties of antireflection coatings. J. Opt. Soc. Am. 52: 437-440.

14.Grebenshchikov I V, Vlasov A G, Neporent B S and Sujkovsky N V. Prosvetlenie Optiki (Non-Reflective Coatings on Optical Materials). Moscow: OGIZ (1946).

15.Dietz N, 2001. Real-time optical characterization of thin film growth. Mat. Sci. Eng. B87: 1-22.

16.Monz'on J J and S'anchez-Soto L L, 1999. Lossless multilayers and Lorentz transformations: more than an analogy. Opt. Commun. 162: 1-6.

17. Thelen A. Design of optical interference coatings. New York: McGraw-Hill (2008).

Анотація. Визначені обвідні функиії спектрів відбивання $i$ пропускання багатошарових структур. 3 допомогою иих обвідних знайдені аналітичні вирази для фазових товщин двох довільних шарів, які забезпечують нульове значення енергетичного коефіцієнта відбивання від всієї прозорої структури. 